\title{
P-selectin glycoprotein ligand-1-deficient mice have impaired leukocyte tethering to E-selectin under flow
}

\author{
Lijun Xia, ${ }^{1}$ Markus Sperandio, ${ }^{2}$ Tadayuki Yago, ${ }^{1}$ J. Michael McDaniel, ${ }^{3}$ \\ Richard D. Cummings, ${ }^{4,5}$ Sonia Pearson-White, ${ }^{6}$ Klaus Ley, ${ }^{2,7}$ and Rodger P. McEver 1,3,4,5 \\ ${ }^{1}$ Warren Medical Research Institute and Department of Medicine, University of Oklahoma Health Sciences Center, \\ Oklahoma City, Oklahoma, USA \\ ${ }^{2}$ Department of Biomedical Engineering, University of Virginia, Charlottesville, Virginia, USA \\ ${ }^{3}$ Cardiovascular Biology Research Program, Oklahoma Medical Research Foundation, Oklahoma City, Oklahoma, USA \\ ${ }^{4}$ Department of Biochemistry and Molecular Biology, and \\ ${ }^{5}$ Oklahoma Center for Medical Glycobiology, University of Oklahoma Health Sciences Center, Oklahoma City, \\ Oklahoma, USA \\ ${ }^{6}$ Department of Microbiology, and \\ ${ }^{7}$ Cardiovascular Research Center, University of Virginia, Charlottesville, Virginia, USA
}

Address correspondence to: Rodger P. McEver, Department of Medicine, University of Oklahoma Health Sciences Center, 825 NE 13th Street, Oklahoma City, Oklahoma 73104, USA.

Phone: (405) 271-6480; Fax: (405) 271-3137; E-mail: rodger-mcever@ouhsc.edu.

Received for publication September 5, 2001, and accepted in revised form February 18, 2002.

P-selectin glycoprotein ligand-1 (PSGL-1) mediates rolling of leukocytes on P-selectin under flow. The glycoproteins that enable leukocyte tethering to or rolling on E-selectin are not known. We used gene targeting to prepare $P S G L$-1-deficient $\left(P S G L-1^{-/}\right)$mice, which were healthy but had moderately elevated total blood leukocytes. Fluid-phase E-selectin bound to approximately $70 \%$ fewer sites on $P S G L-1^{-/}$than $P S G L-1^{+/+}$neutrophils. Compared with $P S G L-1^{+/+}$leukocytes, significantly fewer $P S G L-1^{-/-}$leukocytes rolled on E-selectin in vitro, because their initial tethering to E-selectin was impaired. The residual cells that tethered rolled with the same shear resistance and velocities as $P S G L-1^{+/+}$leukocytes. Compared with $P S G L-1^{+/+}$mice, significantly fewer PSGL-1 $1^{-/-}$leukocytes rolled on E-selectin in TNF- $\alpha$-treated venules of cremaster muscle in which P-selectin function was blocked by an $\mathrm{mAb}$. The residual PSGL-1/-/ leukocytes that tethered rolled with slow velocities equivalent to those of $P S G L-1^{+/+}$leukocytes. These results reveal a novel function for PSGL-1 in tethering leukocytes to E-selectin under flow.

J. Clin. Invest. 109:939-950 (2002). DOI:10.1172/JCI200214151.

\section{Introduction}

Leukocyte recruitment during inflammation is a multistep process involving tethering and rolling of leukocytes on vascular surfaces, followed by firm adhesion and transmigration into the affected tissues (1). Interactions of selectins with glycoconjugate ligands primarily mediate the initial tethering and rolling adhesion of leukocytes $(2,3)$. L-selectin, expressed on leukocytes, binds to ligands on other leukocytes and on activated endothelial cells. P-selectin, expressed on activated platelets and endothelial cells, and E-selectin, expressed on activated endothelial cells, bind to ligands on leukocytes. Studies with selectin-deficient mice and blocking $m A b$ 's indicate that the selectins have both unique and overlapping functions $(4,5)$. In the microcirculation of the cremaster muscle, P-selectin mediates most tethering and rolling of leukocytes early after trauma-induced inflammation (6), whereas L-selectin supports significant tethering and rolling 6-8 hours after exposure to TNF- $\alpha$ (7). E-selectin supports slow rolling of leukocytes on venules $2-4$ hours after stimulation with TNF- $\alpha$ (8).

Selectin ligands require $\alpha 2,3$-sialylation and $\alpha 1,3$ fucosylation on capping structures such as sialyl Lewis $x\left(\mathrm{sLe}^{\mathrm{x}}\right)$, which bind to the C-type lectin domains of the selectins (9). In addition, sulfation is a critical requirement of ligands that bind to P- and L-selectin. A subset of glycoproteins binds with high affinity or avidity to the selectins (2). Of these, only P-selectin glycoprotein ligand-1 (PSGL-1) has a clearly documented function in mediating selectin-dependent cell adhesion under flow $(3,10)$. Human and murine PSGL-1 are extended homodimeric sialomucins that are expressed on most leukocytes. PSGL-1 binds to all three selectins in vitro. $\mathrm{P}$ - and L-selectin bind to the $\mathrm{N}$-terminal region of PSGL-1 (11-13), whereas E-selectin binds to one or more additional sites on PSGL-1 (14). Human PSGL-1 binds to P-selectin with specific stereochemical requirements that include optimal orientation of three tyrosine sulfates, amino acids, and a core-2 O-glycan capped with sLe ${ }^{x}(15-17)$. PSGL-1 does not require tyrosine sulfation to bind to E-selectin, but expression of sLe ${ }^{x}$ on core-2 O-glycans enhances binding (18).

mAb's against the N-terminal region of PSGL-1 markedly inhibit rolling of human and murine leukocytes on P-selectin in vitro $(11,12)$, rolling of human neutrophils on P-selectin in rat venules in vivo (19), and rolling of murine leukocytes on P-selectin in murine venules in vivo $(12,20)$. These data demonstrate that 
PSGL-1 is a dominant ligand for P-selectin under physiological conditions. mAb's against the $\mathrm{N}$-terminal region of murine PSGL-1 also inhibit leukocyte recruitment in some models of inflammation in vivo $(12,21)$. In venules of rats and mice treated with anti-PSGL-1 $\mathrm{mAb}$, a few leukocytes continue to roll on P-selectin at greatly elevated velocities $(12,19)$. Whether this reflects PSGL-1-independent ligands for P-selectin or a failure of the mAb's to block all PSGL-1 molecules has not been established. Furthermore, the mAb's could not be used to establish the contribution of PSGL-1 to rolling on E-selectin, since they do not block binding of purified PSGL-1 to E-selectin in vitro (14).

More recently, gene-targeted mice lacking PSGL-1 (PSGL-1 $1^{-/-}$mice) have been used to study the contributions of PSGL-1 to leukocyte recruitment in vivo (22). The number of rolling leukocytes was markedly decreased in venules of the cremaster muscle 30 minutes after exteriorization, a model of trauma-induced inflammation that is P-selectin dependent. The number of PSGL-1/- leukocytes rolling on venules of TNF- $\alpha$-treated cremaster muscle, which express both P- and E-selectin, was also significantly decreased. However, it was not demonstrated whether this decrease was due solely to impaired rolling on P-selectin or whether PSGL-1 also contributed to tethering to or rolling on E-selectin. Although PSGL-1 and certain other leukocyte glycoproteins bind to E-selectin in solution $(2,9,10)$, no glycoprotein has been definitively shown to mediate leukocyte tethering to or rolling on E-selectin under flow.

Here we report the generation of an independent line of PSGL-1/- mice. Using in vitro and in vivo assays, we demonstrate that PSGL-1 makes a major contribution to leukocyte tethering to E-selectin under flow, but those PSGL-1-1- leukocytes that do tether to E-selectin roll with normal velocities. We also show that ligands other than PSGL-1 enable detectable but unstable rolling of leukocytes on P-selectin.

\section{Methods}

Characterization of murine PSGL-1 genomic structure. A bacterial artificial chromosome (BAC) clone containing the murine PSGL-1 gene was identified by screening a murine embryonic stem cell (ES) BAC library (Incyte Genomics Inc., St. Louis, Missouri, USA) by PCR, using primers derived from the cDNA sequence of murine PSGL-1 (23). Southern blots with probes labeled with $\alpha-{ }^{32}$ P-dCTP (PerkinElmer Life Sciences Inc., Boston, Massachusetts, USA) were used to characterize the structure of the gene. Exon 1, which contains $5^{\prime}$ untranslated sequence, was identified by $5^{\prime}$ RACE PCR using Marathon-Ready cDNA from murine spleen (Clontech Laboratories Inc., Palo Alto, California, USA) as the template. To determine the length of the intron between exons 1 and 2, we amplified its sequence with the Expand Long Template PCR system (Roche Molecular Biochemicals, Indianapolis, Indiana, USA), using the BAC genomic DNA clone as a template, a direct primer complimentary to the $3^{\prime}$ sequence of exon 1 , and a reverse primer complimentary to the $5^{\prime}$ sequence of exon 2. The murine PSGL-1 gene was partially sequenced at the Advanced Center for Genome Technology at the University of Oklahoma.

Targeting vector. Based on Southern blot results, two EcoRI fragments and one BamHI fragment from the murine PSGL-1 gene were subcloned into pBluescript $\mathrm{SK}(-)$ vectors and used for construction of the targeting vector (Figure 1a). A 0.1-kb KpnI-SmaI fragment (from $5 \mathrm{bp}$ upstream of the ATG site to $95 \mathrm{bp}$ downstream of the ATG site of exon 2) (Figure 1, a and b) was replaced by a $1.7-\mathrm{kb}$ KpnI-EcoRV PGK neomycin cassette (a gift from Timothy Ley, Washington University School of Medicine, St. Louis, Missouri, USA). A 1.1-kb region of the $P S G L-1$ gene upstream of the KpnI site was used as a left arm, and a 3.6-kb region downstream of the SmaI site was used as a right arm for homologous recombination (Figure 1, a and b). Both arms were amplified by PCR using Pwo DNA polymerase (Roche Molecular Biochemicals) and 20 amplification cycles to reduce the possibility of mismatches. The fidelity of the construct was confirmed by restriction enzyme mapping and sequencing.

Generation of PSGL-1-deficient mice. Electroporation was used to transfect the targeting vector into CJ7 ES cells (originating in a 129/SvlmJ mouse, a gift from Thomas Sato, University of Texas Southwestern Medical School, Dallas, Texas, USA). After G418 selection, the surviving clones were screened by PCR, and clones with correct homologous recombination were confirmed by Southern blots. After confirming a normal karyotype, ES cells from one of the targeted clones were microinjected into C57BL/6J blastocysts, and the blastocysts were implanted into pseudopregnant mice. Eight chimeras were produced among the offspring. The chimeras were tested for germline transmission by breeding with C57BL/6J mice. The agouti-colored offspring were characterized by both PCR and Southern blot analyses of tail genomic DNA. Heterozygous PSGL-1-deficient $\left(P S G L-1^{+/}\right)$mice were bred to generate homozygous PSGL-1-deficient (PSGL-1-/-) mice. In all experiments reported here, $P S G L-1^{+/+}$littermates were used as controls for PSGL-1/-/ mice.

Flow cytometry. Leukocytes were isolated from heparinized $(10 \mathrm{U} / \mathrm{ml})$ peripheral blood obtained through a carotid artery catheter (see method below), after lysis of erythrocytes in $150 \mathrm{mM} \mathrm{NH}_{4} \mathrm{Cl}, 10 \mathrm{mM}$ $\mathrm{NaHCO}_{3}$, and $1 \mathrm{mM}$ EDTA. Unless otherwise specified, all Ab's were obtained from PharMingen (San Diego, California, USA). To measure expression of PSGL-1, leukocytes in HBSS with 1\% FCS were incubated with phycoerythrin-labeled (PE-labeled) 2PH1, a rat anti-murine PSGL-1 mAb. To examine whether PSGL-1 deficiency affected the expression of other adhesion molecules, leukocytes were incubated with FITC-labeled rat mAb's against murine L-selectin (Mel-14), CD18 (C71/16), CD43 (S7), or CD45 (30-F11). To measure expression of CD24, leukocytes were incu- 
bated with varying concentrations of rat anti-murine CD24 mAb 79 (IgG2a) or M1.69 (IgG2b) (24) (generous gifts from Peter Altevogt, German Cancer Center, Heidelberg, Germany) or with isotype-matched control mAb's. After washing, the cells were incubated with FITC-conjugated goat $\mathrm{F}\left(\mathrm{ab}^{\prime}\right) 2$ anti-rat IgG (Caltag Laboratories Inc., Burlingame, California, USA). To measure binding of fluid-phase selectins, leukocytes were incubated with murine P- or E-selectin-IgM chimeras or a control CD45-IgM chimera (25). The chimeras were obtained from conditioned medium of COS-7 cells that were transfected with pCDM8 vectors encoding each molecule (generous gifts from John B. Lowe, University of Michigan Medical Center, Ann Arbor, Michigan, USA). Binding of the selectin chimeras was detected by incubation with FITC-conjugated goat anti-human IgM polyclonal Ab's (Chemicon International, Temecula, California, USA). Serial dilutions of conditioned medium were added to determine the concentrations required for saturable selectin binding to cells. All data shown represent binding at selectin-IgM concentrations that were at least twice those required to saturate binding sites on leukocytes. Binding specificity was confirmed by parallel incubations in buffer containing $10 \mathrm{mM}$ EDTA or by preincubation of the respective selectin chimera with 20 $\mu \mathrm{g} / \mathrm{ml}$ anti-murine P-selectin mAb RB40.34 (6) or anti-murine E-selectin mAb 9A9 (a gift from Barry Wolitzky, MitoKor, San Diego, California, USA) (26). For all analyses, leukocytes were pretreated with 20 $\mu \mathrm{g} / \mathrm{ml}$ mouse Fc Block (PharMingen) for 20 minutes at $4^{\circ} \mathrm{C}$ or with $20 \mu \mathrm{g} / \mathrm{ml} \mathrm{Fc}$ Receptor Blocker (Accurate
Chemical \& Scientific Corp., Westbury, New York, USA) for 20 minutes at room temperature. Neutrophils were identified using FITC-labeled RB6-8C5 $\mathrm{mAb}$ against the myeloid differentiation antigen Ly-6G (Gr-1), or by the light scatter properties of neutrophils. Cells were analyzed on a FACScan machine using CellQuest software (Becton Dickinson Immunocytometry Systems, San Jose, California, USA).

Hematology and histology. Peripheral blood was collected from the mouse tail vein using Drummond Microcaps (Drummond Scientific Co., Broomall, Pennsylvania, USA). Total leukocyte numbers were counted in a hemocytometer by diluting $10 \mu \mathrm{l}$ blood in $90 \mu \mathrm{l} \mathrm{Kimura} \mathrm{stain} \mathrm{consisting} \mathrm{of} 11 \mathrm{ml}$ of $5 \%$ (wt/wt) toluidine blue, $0.8 \mathrm{ml}$ of $0.03 \%$ light green SF yellowish, $0.5 \mathrm{ml}$ of saturated saponin, and $5 \mathrm{ml}$ of $0.07 \mathrm{M}$ phosphate buffer, pH 6.4, all from Sigma Chemical Co. (St. Louis, Missouri, USA). Differential leukocyte counts were obtained from Wright-stained blood smears. Platelet and hemoglobin levels were measured with a Serono 9000 cell counter (Serono-Parker Diagnostics Inc., Allentown, Pennsylvania, USA). For histological analysis, organs from PSGL-1//+ and PSGL-1-1littermates were fixed in $10 \%$ formalin, processed, and embedded in paraffin. Tissue sections were stained with hematoxylin and eosin.

In vitro flow chamber assay. $\mathrm{MH} 15-1$, an $\mathrm{mAb}$ against the Fc portion of human IgM $(20 \mu \mathrm{g} / \mathrm{ml}$; Accurate Chemical \& Scientific Corp.) was coated on $35-\mathrm{mm}$ polystyrene dishes in sodium carbonate buffer ( $\mathrm{pH}$ 9.6) overnight at $4{ }^{\circ} \mathrm{C}$. The dishes were washed three times with HBSS containing $0.1 \%$ human serum albumin

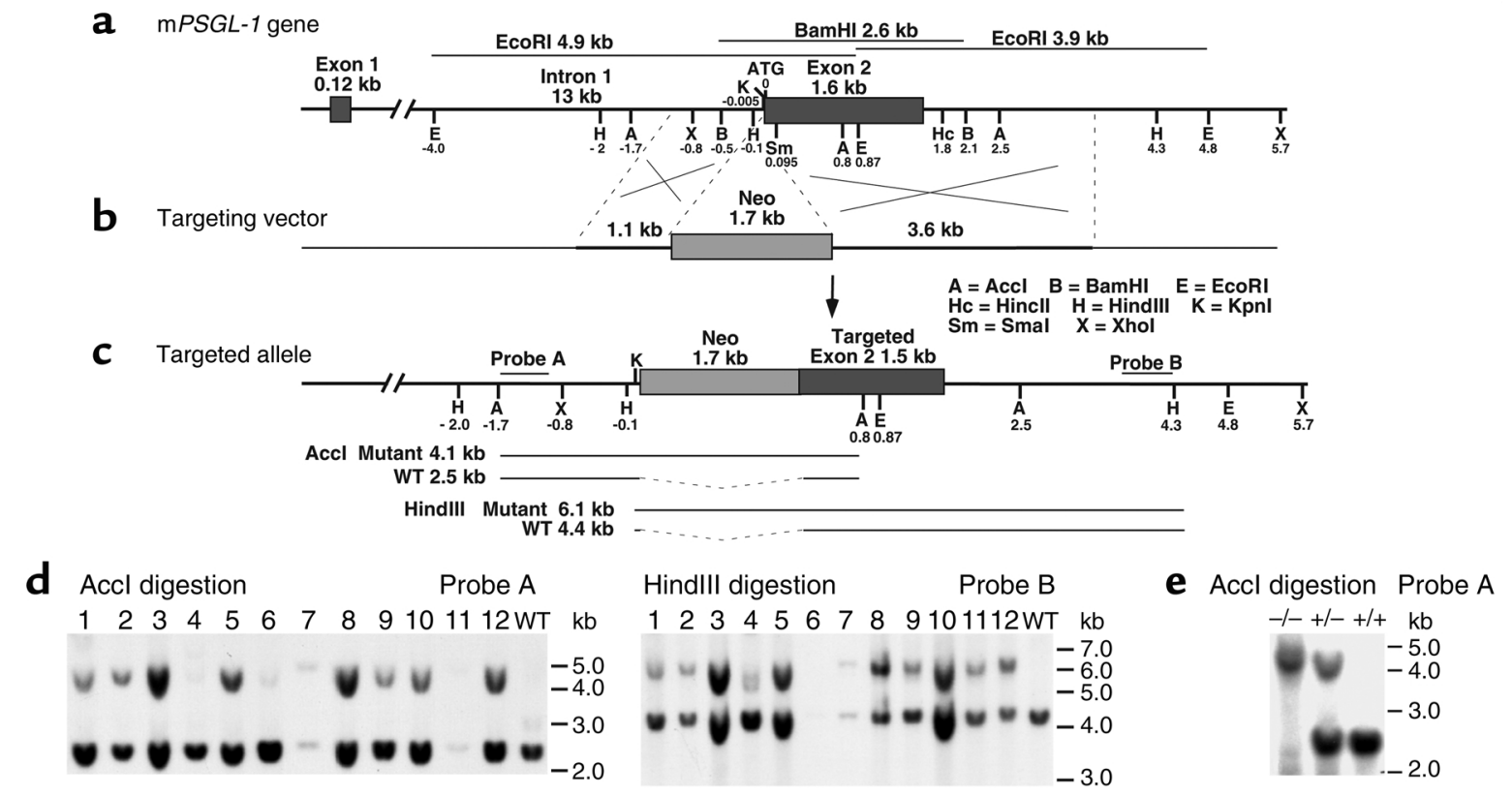

\section{Figure 1}

Strategy for disrupting the murine PSGL-1 gene. (a) Partial restriction map of the murine PSGL-1 gene. The three restriction fragments used for construction of the targeting vector are shown at the top. (b) Diagram of the targeting vector. (c) Diagram of the targeted gene, the probes used for Southern blotting, and the predicted sizes of the restriction fragments for the WT and targeted alleles. (d) Southern blots of genomic DNA from targeted and WT ES cells. (e) Southern blots of genomic DNA isolated from tails of PSGL-1 $1^{+/+}, P S G L-1^{+/-}$, and $P S G L-1^{-/-}$mice. 
(HSA), blocked with 1\% HSA in HBSS for 2 hours, and incubated with P- or E-selectin-IgM chimera for 1 hour at $37^{\circ} \mathrm{C}$. Site densities of mAb-captured P- or E-selectin-IgM were measured by binding of ${ }^{125}$ I-labeled anti-P-selectin mAb RB40.34 or anti-E-selectin $\mathrm{mAb}$ 10E9.6 (PharMingen) as described (14). Heparinized blood obtained through a carotid artery catheter was incubated with ammonium chloride to lyse erythrocytes. It was then diluted in HBSS containing $0.5 \%$ HSA and mixed gently with 4-mm glass beads (Fisher Scientific Co., Pittsburgh, Pennsylvania, USA) to remove platelets. After removing the beads, the leukocytes were suspended at $10^{6}$ cells $/ \mathrm{ml}$ in HBSS with $0.5 \%$ HSA. The cells were perfused over P- or E-selectin substrates in dishes mounted in a parallel-plate flow chamber at wall shear stresses ranging from 0.25 to $4 \mathrm{dyn} / \mathrm{cm}^{2}$. After 5 minutes, the accumulated number of rolling cells was measured using a videomicroscopy system coupled to a digital analysis system (Inovision, Cleveland, Ohio, USA) on a Silicon Graphics workstation (27). In some experiments, $10 \mathrm{mM}$ EDTA, $4 \mu \mathrm{g} / \mathrm{ml} \mathrm{F}\left(\mathrm{ab}^{\prime}\right) 2$ fragments of Mel-14, $20 \mu \mathrm{g} / \mathrm{ml}$ anti-PSGL-1 mAb 4RA10 (a gift from Dietmar Vestweber, University of Münster, Münster, Germany), $20 \mu \mathrm{g} / \mathrm{ml}$ anti-CD2 $4 \mathrm{mAb} 79$ or M1.69, or an equivalent concentration of isotype-matched control $\mathrm{mAb}$ was added to the cells. In other experiments, the dishes were preincubated with $20 \mu \mathrm{g} / \mathrm{ml}$ anti-E-selectin mAb 9A9, anti-P-selectin mAb RB40.34, or isotype-matched control mAb's. The cells were perfused through the chamber in the continued presence of the antibody. In some experiments, PSGL-1-1- leukocytes were preincubated in $0.5 \mathrm{ml} \mathrm{HBSS}$ with $0.5 \% \mathrm{HSA}$ for 1 hour at $37^{\circ} \mathrm{C}$ in the presence or absence of Pronase $\left(1 \mathrm{mg} / 10^{6}\right.$ leukocytes; CalbiochemNovabiochem Corp., San Diego, California, USA) or O-sialoglycoprotein endopeptidase (OSGE, $250 \mu \mathrm{g} / 10^{6}$ leukocytes; Cedarlane Laboratories Ltd., Hornby, Ontario, Canada). The cells were washed twice and then perfused over P-selectin substrates. Flow cytometry revealed that OSGE treatment removed epitopes for the mucin CD43 but not for the non-mucin glycoprotein CD18. In contrast, Pronase removed epitopes for both proteins from leukocyte surfaces.

To measure resistance to detachment under shear, PSGL-1 $1^{+/+}$leukocytes $\left(10^{6} / \mathrm{ml}\right)$ or $P S G L-1^{-/-}$leukocytes $\left(4 \times 10^{6} / \mathrm{ml}\right)$ were allowed to accumulate at 0.5 or 0.25 $\mathrm{dyn} / \mathrm{cm}^{2}$, and then cell-free buffer was introduced. Wall shear stress was increased every 30 seconds, and the percentage of cells remaining adherent was determined. The velocities of rolling cells were measured over a 5 -second interval (14). The rate at which flowing cells became tethered to P- or E-selectin was measured during the first 60 seconds of perfusion. For tether measurements, E-selectin was captured in a small area in the middle of the dish, and then the rest of the dish was blocked with $1 \%$ HSA in HBSS. Tethers to E-selectin were measured near the upstream junction with the blocked surface. Thus, only flowing leukocytes directly tethering to E-selectin (primary tethers) were measured, rather than secondary tethers to E-selectin that occurred after initial L-selectin-dependent tethering of flowing leukocytes to adherent leukocytes (13, 28). The tethering rate at different wall shear stresses was normalized by dividing the number of tethered cells by the number of cells transported across the field of view in the focal plane of the substrate (27).

In some experiments, interactions of flowing PSGL-1/++ or PSGL-1//- leukocytes with an L-selectin ligand and with murine VCAM-1 were measured. The L-selectin ligand was 2-GSP-6, a glycosulfopeptide modeled after the $\mathrm{N}$-terminal P-selectin-binding region of human PSGL-1, which also binds to human and murine L-selectin (16). Streptavidin $(50 \mu \mathrm{g} / \mathrm{ml})$ adsorbed onto $35-\mathrm{mm}$ dishes was used to capture 2-GSP-6 that was biotinylated through a C-terminal cysteine (T. Yago et al., unpublished observations). Site densities of 2-GSP- 6 were measured by the binding of ${ }^{125}$ I-labeled anti-human PSGL-1 mAb PL1 (11). Murine VCAM-1 $(10 \mu \mathrm{g} / \mathrm{ml})$, expressed as a recombinant protein fused to the $\mathrm{Fc}$ portion of human IgG (R\&D Systems Inc., Minneapolis, Minnesota, USA), was adsorbed directly onto $35-\mathrm{mm}$ dishes, and the dishes were then blocked with 1\% HSA in HBSS. The accumulated number of rolling leukocytes and the rate at which flowing leukocytes tethered to 2-GSP-6 or VCAM-1 were measured as for P- or E-selectin. The specificity of the interactions was confirmed by demonstrating that anti-murine L-selectin $\mathrm{mAb}$ Mel-14 blocked tethering and rolling on 2-GSP-6 and that anti-murine VCAM-1 mAb MK-1 (29) (a gift from Paul Kincade, Oklahoma Medical Research Foundation, Oklahoma City, Oklahoma, USA) blocked tethering and rolling on VCAM-1.

Intravital microscopy. Mice were anesthetized with an intraperitoneal injection of ketamine (Ketalar, 125 $\mathrm{mg} / \mathrm{g}$ body wt; Parke-Davis, Detroit, Michigan, USA) and xylazine (12.5 mg/g body wt; Phoenix Scientific Inc., San Marcos, California, USA), and placed on a heating pad to maintain body temperature. Intravital microscopy was conducted using a microscope (Axioskop; Carl Zeiss Inc., Thornwood, New York, USA) with a saline immersion $\times 40$ objective with a 0.75 numerical aperture. The microscope was equipped with a charge-coupled device (CCD) camera (model VE1000-CCD; Dage-MTI Inc., Michigan City, Indiana, USA), which was connected to a video recorder (Panasonic, Osaka, Japan). After tracheal intubation, the left carotid artery was cannulated for systemic administration of anesthetic and mAb's during the intravital microscopy experiment. The cremaster muscle was prepared as described (8), exteriorized, and superfused with thermocontrolled $\left(35^{\circ} \mathrm{C}\right)$ bicarbonate-buffered saline. Systemic blood samples $(10 \mu \mathrm{l})$ were taken via the carotid artery catheter after each mAb injection, to assess systemic leukocyte counts using the Kimura stain. Leukocyte rolling was observed in venules with diameters ranging from $15 \mu \mathrm{m}$ to $50 \mu \mathrm{m}$. In some experiments the following $\mathrm{mAb}$ 's were injected: anti-murine E-selectin mAb 9A9 (rat IgG1, 30 
$\mu \mathrm{g} /$ mouse), anti-murine P-selectin mAb RB40.34 (rat IgG1, $30 \mu \mathrm{g} /$ mouse), anti-murine L-selectin mAb Mel14 [rat IgG2a F( $\left.\mathrm{ab}^{\prime}\right) 2$ fragments, $30 \mu \mathrm{g} / \mathrm{mouse}$, or isotype control rat monoclonal IgG1 $(30 \mu \mathrm{g} /$ mouse) or IgG2a (30 $\mu \mathrm{g} /$ mouse). Recombinant murine TNF- $\alpha$ (500 ng per mouse; R\&D Systems Inc.) was diluted in $0.3 \mathrm{ml}$ sterile saline and injected intrascrotally 2 hours before the beginning of each experiment. Microvessel diameters, lengths, and rolling leukocyte velocities were measured using a digital image processing system (30). Each rolling leukocyte passing a line perpendicular to the vessel axis was counted, and leukocyte rolling flux was expressed as leukocytes per minute. Rolling flux fraction was calculated as described (6) by dividing leukocyte rolling flux by the total leukocyte flux, which was estimated as [WBC] $\times v_{\mathrm{b}} \times \pi \times(d / 2)^{2}$, where WBC is the systemic leukocyte count, $v_{\mathrm{b}}$ is the mean blood flow velocity, and $d$ is the venular diameter. Leukocyte rolling velocities (5-10 leukocytes per venule) were measured as averages over a period of 2 seconds. Centerline blood flow velocity was measured using a dual photodiode and a digital online cross-correlation program (CircuSoft Instrumentation LLC, Hockessin, Delaware, USA) and converted to mean blood flow velocity by multiplying by an empirical factor of 0.625 (31). Wall shear rates $\left(\gamma_{\mathrm{w}}\right)$ were estimated as $2.12\left(8 v_{\mathrm{b}} / d\right)$, where $v_{\mathrm{b}}$ is the mean blood flow velocity, $d$ is the diameter of the vessel, and 2.12 is a median empirical correction factor obtained from velocity profiles measured in microvessels in vivo (32).

Data analysis of the intravital microscopy experiments. Statistical analysis was performed using Sigma-Stat 2.0 software (SPSS Science, Chicago, Illinois, USA). Average vessel diameter, leukocyte rolling flux fractions, leukocyte rolling velocities, and wall shear rates between groups and treatments were compared with the one-way ANOVA on ranks (Kruskal-Wallis) with a multiple pairwise comparison test (Dunn's test). Rolling flux fractions between untreated and mAbtreated groups were compared using the Student $t$ test or the Wilcoxon rank-sum test as appropriate. Statistical significance was set at $P<0.05$, as indicated by an asterisk in the figures.

\section{Results}

Characterization of PSGL-1 gene structure. The murine PSGL-1 gene was previously shown to contain a $1.6-\mathrm{kb}$ exon containing the entire open reading frame plus the 3 ' untranslated sequence of the murine PSGL-1 cDNA $(22,23)$. However, this exon, here termed exon 2 , did not contain most of the $5^{\prime}$ untranslated sequence. We used $5^{\prime}$ RACE PCR of murine spleen cDNA to identify the remainder of the $5^{\prime}$ untranslated region. This sequence was found to reside in a $0.12-\mathrm{kb}$ exon, here termed exon 1. A PCR procedure optimized for amplification of long sequences revealed that the intron connecting exons 1 and 2 is approximately $13 \mathrm{~kb}$ long (Figure 1a). Thus, the structure of the murine PSGL-1 gene resembles that of the human $P S G L-1$ gene, which was previously shown to have a short exon encoding the $5^{\prime}$ untranslated sequence, a long intron, and a second exon containing the entire open reading frame and $3^{\prime}$ untranslated sequence (33). Based on Southern blot analysis, we subcloned two EcoRI fragments and one BamHI fragment, which cover most of the murine gene, into pBluescript $\mathrm{SK}(-)$ vectors (Figure 1a). Sequencing of these clones revealed some AT- and CTrich sequences located upstream and downstream of exon 2, as previously reported (22).

Generation of PSGL-1-deficient mice. A targeting vector was prepared that replaces the $5^{\prime}$ portion of exon 2 , including the ATG translational start site, with a neomycin-resistance gene cassette (Figure 1, b and c). After transfection of ES cells with the targeting vector, 880 G418-resistant clones were screened by PCR, and 12 candidate targeted clones were identified. These clones were subjected to Southern blot analysis to confirm those with correct homologous recombination (Figure 1d). Probe A detected a $2.5-\mathrm{kb}$ AccI fragment from the wild-type (WT) allele and a 4.1-kb AccI fragment from the mutant allele, whereas probe $\mathrm{B}$ detected a 4.4-kb HindIII fragment from the wildtype allele and a 6.1-kb HindIII fragment from the mutant allele. Random integration of extra copies of the targeting vector was excluded by hybridization with a neomycin cDNA probe (data not shown). Contamination with WT ES cells was excluded by choosing clones in which hybridization revealed equally intense WT and mutant bands. Correct homologous recombination was confirmed in ES clones 3, 7, 8, and 12 (Figure 1d). Unlike a previously reported study (22), we did not need to delete repetitive sequences from the arms of our targeting vector to obtain correct homologous recombination. We microinjected cells from clone 3 into blastocysts, and implanted the blastocysts into pseudopregnant mice. Eight chimeric mice were produced, and the four chimeras that were bred all transmitted the targeted PSGL-1 allele to their offspring. The PSGL-1+/- mice were bred to generate homozygous PSGL-1-1- mice in a mixed 129/SvlmJ and C57BL/6J genetic background. Southern blots confirmed the disruption of the PSGL-1 gene (Figure 1e).

General analysis of PSGL-1-deficient mice. To confirm that the gene disruption eliminated expression of PSGL-1 in leukocytes, we used flow cytometry to measure binding of PE-labeled anti-PSGL-1 mAb 2PH1 to neutrophils that were identified by an FITClabeled $\mathrm{mAb}$ against Ly-6G (Gr-1). 2PH1 bound to $P S G L-1^{+/+}$neutrophils and (to a slightly lesser extent) to heterozygous $P S G L-1^{+/-}$neutrophils. However, 2PH1 did not bind to $P S G L-1^{-/-}$neutrophils, confirming the null mutation (Figure 2). FITC-conjugated mAb's against CD18, CD43, CD45, and L-selectin bound equivalently to $P S G L-1^{+/+}$and $P S G L-1^{-/-}$neutrophils, indicating that deficiency of PSGL-1 did not indirectly affect the expression of other major cell-surface glycoproteins (data not shown). 

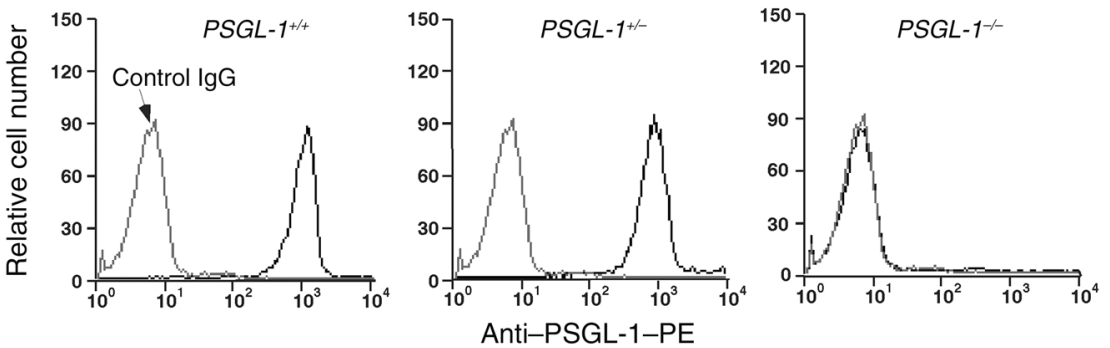

Figure 2
Flow cytometric analysis of PSGL-1
expression on peripheral blood neu-
trophils from $P S G L-1^{+/+}, P S G L-1^{+/-}$, and
$P S G L-1^{-/-}$mice. The cells were incubated
with PE-conjugated rat control IgG1 or
with PE-conjugated rat anti-murine
PSGL-1 mAb $2 \mathrm{PH} 1$. The data are repre-
sentative of more than ten experiments.

PSGL-1/- mice developed normally, and both sexes were fertile. Crosses between heterozygotes yielded normal-sized litters. Among 321 offspring (158 males and 163 females), $31 \%$ were $P S G L-1^{+/+}, 42 \%$ were $P S G L-1^{+/-}$, and $27 \%$ were $P S G L-1^{-/-}$, indicating normal Mendelian inheritance. Unlike mice deficient in both P- and E-selectin $(34,35), P S G L-1^{-/}$mice remained free of mucocutaneous infections to at least 5 months of age. Thus, the general development of our PSGL-1 ${ }^{-/-}$mice resembled that of previously generated $P S G L-1^{-/-}$mice (22). The latter mice were reported to have normal total leukocyte counts, but slightly elevated neutrophil counts in peripheral blood, much like that of P-selectin $/-$ mice $(36,37)$. The PSGL-1-1- mice that we generated had moderately elevated total leukocyte counts with significant increases in neutrophils, lymphocytes, and eosinophils (Table 1). Hemoglobin and platelet levels were similar in PSGL-1 $1^{+/+}$and PSGL-1/- mice. Histological examination of multiple organs, including spleen, lymph node, Peyer's patch, and thymus, revealed no differences between PSGL-1//+ and PSGL-1//- mice.

Binding of selectin-IgM chimeras to lenkocytes. We used flow cytometry to compare the binding of murine P- and E-selectin-IgM chimeras to $P S G L-1^{+/+}$and PSGL-1/-- neutrophils. As previously reported (25), P-selectin-IgM and E-selectin-IgM each bound to PSGL-1/++ neutrophils (Figure 3, a and b). Binding was specific, because a control CD45-IgM chimera did not bind, and addition of anti-P-selectin or antiE-selectin $\mathrm{mAb}$ blocked binding of the respective selectin. EDTA also blocked binding, consistent with the $\mathrm{Ca}^{2+}$-dependent nature of selectin-ligand interactions (data not shown). P-selectin-IgM did not detectably bind to $P S G L-1^{-/-}$neutrophils (Figure 3c). Thus, no PSGL-1-independent ligands for P-selectin on neutrophils could be identified in this assay. E-selectin-IgM bound to PSGL-1 $1^{-/-}$neutrophils, but the binding was consistently reduced to about $30 \%$ of that observed on $P S G L-1^{+/+}$neutrophils (Figure $3 \mathrm{~d}$ ). These data demonstrate that PSGL-1 accounts for many, but not all, of the ligands for E-selectin that are measured in this assay.

Tethering and rolling ofleukocytes on E- and P-selectin in vitro. We compared the abilities of PSGL-1//+ and PSGL-1//leukocytes to tether to and roll on immobilized E- and P-selectin-IgM under shear flow. PSGL-1//+ leukocytes rolled specifically on E- and P-selectin, since rolling was blocked by the addition of anti-selectin mAb's (Figure 4a and Figure 5a) but not by isotype-matched control mAb's (data not shown).

PSGL-1 $1^{-/-}$leukocytes rolled on E-selectin, but significantly fewer $P S G L-1^{-/-}$than $P S G L-1^{+/+}$leukocytes rolled on E-selectin at all wall shear stresses examined (Figure 4 , $a$ and $b$ ). The residual rolling of $P S G L-1^{-/-}$leukocytes on E-selectin was specific, because it was blocked by anti-E-selectin mAb 9A9 but not by an isotype-matched control $\mathrm{mAb}$ (Figure $4 \mathrm{a}$ and data not shown). A rolling leukocyte can be recruited directly through tethering of a flowing cell to E-selectin (primary tether) or indirectly through $\mathrm{L}$-selectin-dependent tethering of a flowing cell to an adherent leukocyte, followed by transfer of the cell from the adherent leukocyte to the E-selectin substrate (secondary tether) (28). PSGL-1/++ leukocytes rolling on E-selectin formed clusters and strings, a pattern characteristic of recruitment by both primary and secondary tethers. In contrast, PSGL-1 $1^{-/-}$leukocytes rolled in a dispersed pattern typical of recruitment solely by primary tethers (data not shown). Anti-L-selectin $\mathrm{mAb}$ Mel-14, but not an isotype-matched control mAb, eliminated clusters and strings, and partially reduced the number of $P S G L-1^{+/+}$cells rolling on E-selectin. In contrast, the anti-L-selectin $\mathrm{mAb}$ had no effect on the pattern or the number of PSGL-1/- cells rolling on E-selectin (Figure $4 \mathrm{~b}$ and data not shown). This result confirms previous conclusions, based on use of anti-PSGL-1 mAb's, that PSGL-1 is the predominant ligand mediating L-selectin-dependent leukocyteleukocyte interactions under flow $(13,38)$. Even when L-selectin function was blocked, significantly fewer PSGL-1-/- than PSGL-1 $1^{+/+}$leukocytes rolled on E-selectin (Figure 4b). This reduced number of rolling leukocytes resulted from an impaired ability of flowing PSGL-1 $1^{-/-}$

\section{Table 1}

Peripheral blood counts

\begin{tabular}{lcc}
\hline Blood cell type & $P S G L-1^{+/+}$ & $P S G L-1^{-/-}$ \\
Total leukocytes $($per $\mu \mathrm{l})$ & $5,900 \pm 600(16)$ & $9,600 \pm 600^{\mathrm{A}}(33)$ \\
Neutrophils & $570 \pm 80(13)$ & $2,400 \pm 130^{\mathrm{A}}(30)$ \\
Lymphocytes & $5,100 \pm 110(13)$ & $6,600 \pm 150^{\mathrm{A}}(30)$ \\
Monocytes & $190 \pm 40(13)$ & $220 \pm 40(30)$ \\
Eosinophils & $80 \pm 20(13)$ & $420 \pm 60^{\mathrm{B}}(30)$ \\
Platelets $\left(\times 10^{-3} / \mu \mathrm{l}\right)$ & $1,200 \pm 60(11)$ & $1,100 \pm 50(7)$ \\
Hemoglobin $(\mathrm{g} / \mathrm{dl})$ & $16.0 \pm 0.7(10)$ & $17.0 \pm 1.0(8)$
\end{tabular}

Data are presented as mean \pm SEM. The number of mice is shown in parentheses. ${ }^{A} P<0.001,{ }^{\mathrm{B}} P<0.005$ vs. $P S G L-1^{+/+}$according to Student $t$ test. 

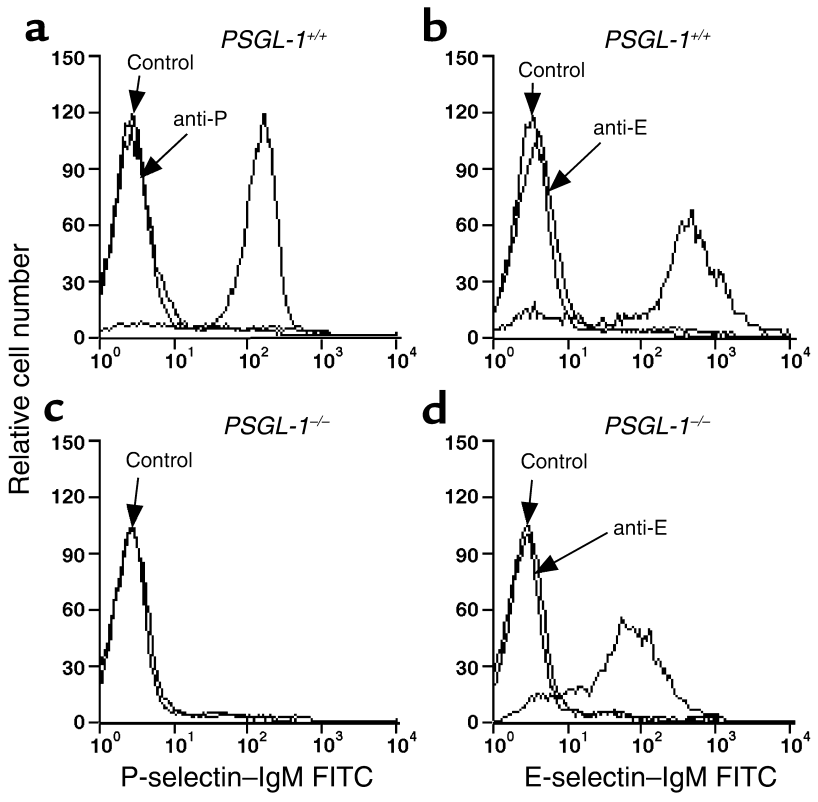

cells to tether to E-selectin, measured under conditions where only primary tethers were observed (Figure 4c). The tethering defect was specific for E-selectin, because flowing PSGL-1/++ and PSGL-1-1/- leukocytes tethered at identical rates to 2-GSP-6, a glycosulfopeptide ligand for L-selectin, and to VCAM-1, a ligand for leukocyte $\alpha 4$ integrins (data not shown). After tethering, PSGL-1//leukocytes and $P S G L-1^{+/+}$leukocytes rolled equivalently on E-selectin. PSGL-1 $1^{-/-}$and PSGL-1//+ leukocytes resisted detachment similarly and rolled with comparable mean velocities as wall shear stress was increased (Figure 4, $\mathrm{d}$ and e). Frame-by-frame analysis revealed that PSGL-1/-/ and PSGL-1/+/ leukocytes manifested similar smooth rolling, and the variances of rolling velocities were similar for leukocytes from both types of mice (data not shown). These results demonstrate that PSGL-1 contributes significantly to the initial tethering

\section{Figure 4}

Tethering and rolling of PSGL-1+/+ and PSGL-1 ${ }^{-/-}$leukocytes on E-selectin in vitro. (a) Leukocytes were perfused over mAb-captured E-selectin-IgM or capture mAb only, in the presence or absence of EDTA or anti-E-selectin mAb 9A9. After 5 minutes, the number of rolling cells was quantified. ${ }^{*} P<0.05$ vs. $P S G L-1^{+/+}$. (b) Leukocytes in the presence or absence of anti-L-selectin mAb Mel-14 were perfused over E-selectin-IgM at the indicated wall shear stress. After 5 minutes, the number of rolling cells was quantified. ${ }^{*} P<0.05$ vs. PSGL-1+/+ + anti-L. (c) Leukocytes were perfused over E-selectin-IgM at the indicated wall shear stress. The number of cells that tethered to E-selectin during the first 30 seconds was quantified and normalized by dividing by the number of cells delivered across the field of view in the focal plane of the monolayer. Only primary tethers of flowing leukocytes to E-selectin were measured. ${ }^{*} P<0.05$ vs. PSGL-1+/+. (d and e) Leukocytes were allowed to accumulate on E-selectin under a wall shear stress of $0.5 \mathrm{dyn} / \mathrm{cm}^{2}$. Wall shear stress was increased every 30 seconds, and the percentage of cells remaining adherent and the rolling velocities of the cells were determined. Data represent mean \pm SEM of four experiments.

\section{Figure 3}

Flow cytometric analysis of binding of P-selectin-IgM and E-selectin-IgM to neutrophils from PSGL-1+/+ and PSGL-1-/- mice. The cells were incubated with saturating concentrations of P-selectin-IgM (a and $\mathbf{c}$ ), E-selectin-IgM (b and $\mathbf{d}$ ), or control CD45-IgM in the presence or absence of anti-P-selectin mAb RB40.34 (anti-P) or anti-E-selectin mAb 9A9 (anti-E). Binding was detected with FITC-conjugated goat anti-human IgM polyclonal $A b$ 's. The data are representative of four experiments.

of leukocytes to E-selectin. After tethering, however, PSGL-1 does not detectably contribute to stable leukocyte rolling on E-selectin.

Virtually no PSGL-1/- leukocytes rolled on moderate densities of P-selectin (Figure 5a). At very high P-selectin densities, a very small number of $P S G L-1^{-/-}$ leukocytes rolled at a very low wall shear stress $(0.25$ dyn $\left./ \mathrm{cm}^{2}\right)$; this rolling was specific, since an antiP-selectin $\mathrm{mAb}$, but not an isotype-matched control $\mathrm{mAb}$, eliminated the rolling (Figure $5 \mathrm{~b}$ and data not shown). The residual rolling was not due to a compensatory increase in P-selectin ligands in the PSGL-1/leukocytes, because similar residual rolling was observed for $P S G L-1^{+/+}$leukocytes treated with anti-PSGL-1 mAb 4RA10 (Figure 5b). Compared with PSGL-1 ${ }^{+/+}$leukocytes, the PSGL-1/- leukocytes that tethered at low wall shear stress detached rapidly as wall shear stress was increased, and rolled with rapidly increasing velocities until they detached (Figure 5, c

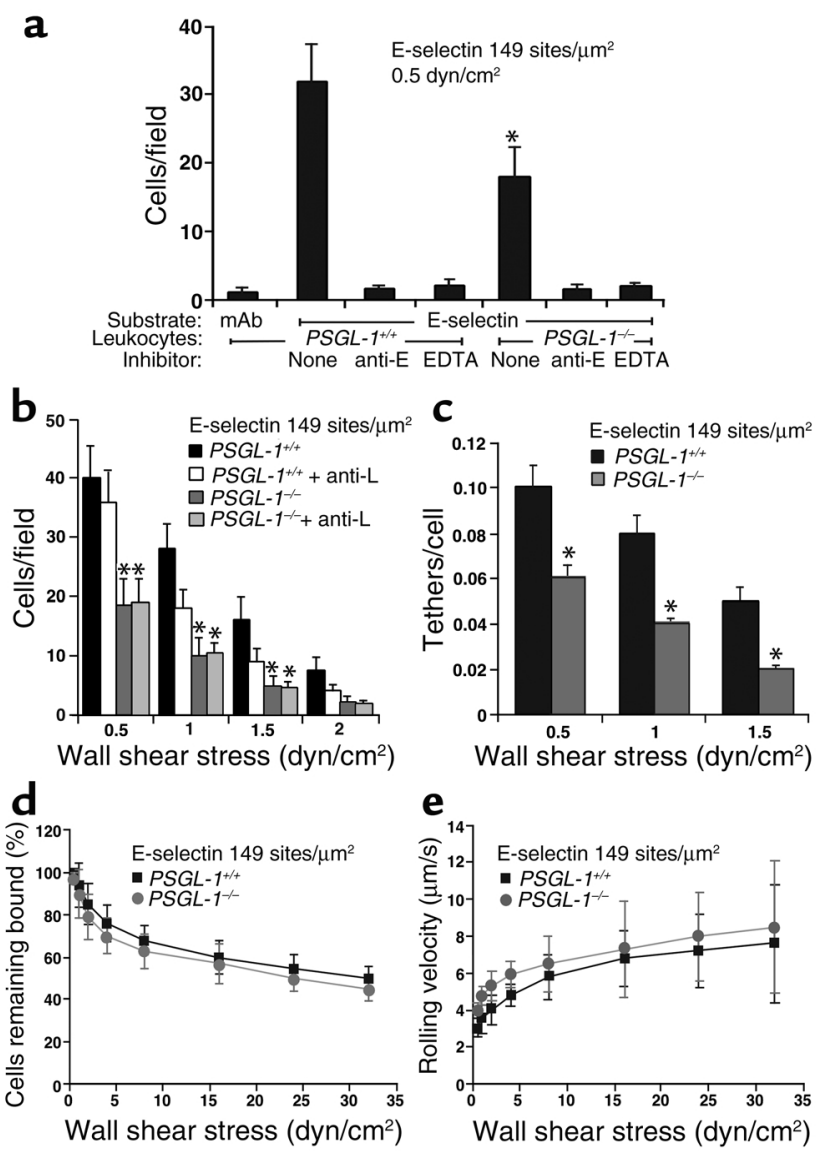




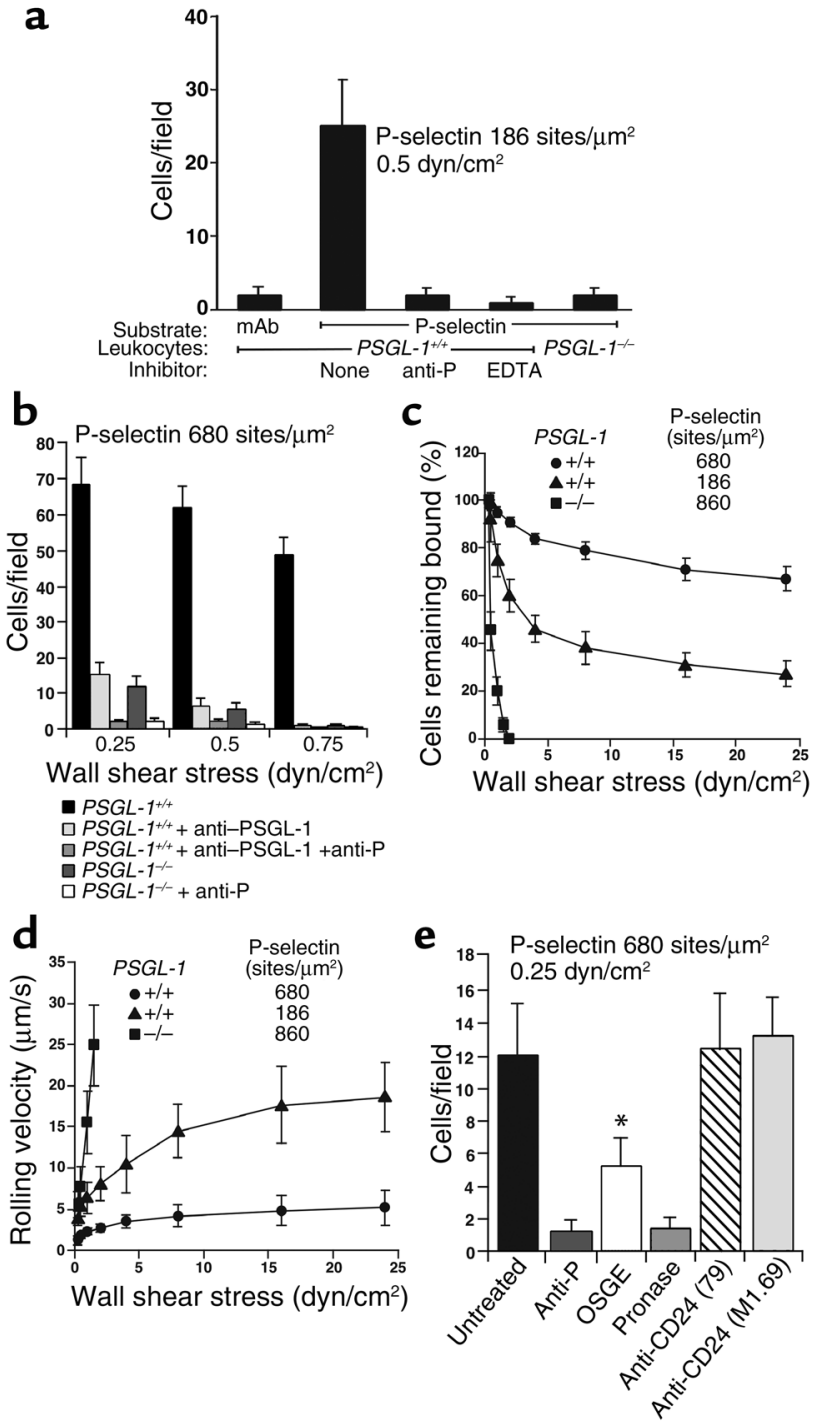

and d). Pretreatment of PSGL-1/-- leukocytes with Pronase, a mixture of many proteases, eliminated the residual rolling on P-selectin, and pretreatment with OSGE, which cleaves proteins with clustered O-glycans (39), decreased rolling by approximately $50 \%$ (Figure 5e). These data suggest that the PSGL-1-independent ligands for P-selectin comprise one or more proteins, at least one of which has mucin characteristics. CD24, a heavily O-glycosylated protein on murine leukocytes, has been shown to bind to P-selectin (24). However, incubation of $P S G L-1^{-1-}$ leukocytes with two well-characterized anti-CD24 mAb's that inhibit most or all binding of P-selectin to purified CD24 (24) had no effect on the rolling of PSGL-1/- leukocytes on P-selectin (Figure 5e).

Lenkocyte rolling in venules with TNF- $\alpha$-induced inflammation. We studied leukocyte rolling in venules of the cremaster muscle 2 hours after stimulation with TNF- $\alpha$, which induces the expression of E-selectin and enhances the expression of P-selectin. Leukocytes roll on both selectins in this model (40). In venules of the size

\section{Figure 5}

Rolling of PSGL-1+/+ and PSGL-1-/- leukocytes on P-selectin in vitro. (a) Peripheral blood leukocytes were perfused over P-selectin-IgM captured on adsorbed $m A b$ against the $F c$ portion of human IgM or over adsorbed $\mathrm{mAb}$ alone, in the presence or absence of EDTA or antiP-selectin mAb RB40.34. After 5 minutes, the number of rolling cells was quantified. (b) Leukocytes were perfused over a very high density of P-selectin-IgM at the indicated wall shear stress in the presence or absence of anti-PSGL-1 mAb 4RA10 and/or anti-P-selectin mAb RB40.34. After 5 minutes, the number of rolling cells was quantified. (c and d) Leukocytes were allowed to accumulate on P-selectin at 0.5 dyn $/ \mathrm{cm}^{2}$. Wall shear stress was increased every 30 seconds, and the percentage of cells remaining adherent and the rolling velocities of the cells were determined. (e) PSGL-1-1- leukocytes preincubated with or without Pronase, OSGE, or anti-CD24 mAb 79 or M1.69 were perfused over high density P-selectin in the presence or absence of anti$\mathrm{P}$-selectin mAb RB40.34. After 5 minutes, the number of rolling cells was quantified. The data in each panel represent the mean \pm SEM of three or four experiments. ${ }^{*} P<0.05$ vs. untreated $P S G L-1^{-1-}$.

and wall shear rate studied, there are virtually no L-selectin-dependent leukocyte-leukocyte interactions $(8,20,41)$. We compared leukocyte rolling in 42 venules of 10 TNF- $\alpha$-treated $P S G L-1^{-1-}$ mice with rolling in 38 venules of 11 TNF- $\alpha$-treated PSGL-1 $1^{+/+}$littermate controls. Hemodynamic and microvascular parameters for both groups showed no significant differences in vessel diameter, blood flow velocity, or wall shear rate (Table 2). Leukocyte rolling was assessed as leukocyte rolling flux fraction, which is defined as the number of rolling leukocytes divided by the total number of leukocytes passing through the same vessel (42). Consistent with previous data (22), the leukocyte rolling flux fraction was reduced to $7 \%$ in $P S G L-1^{-/-}$mice compared with $19 \%$ in $P S G L-1^{+/+}$ mice $(P<0.05)$ (Figure 6a). In the earlier study, the reduction in rolling flux was ascribed solely to defective leukocyte adhesion to P-selectin, although interactions with E-selectin were not directly measured (22).

To determine whether PSGL-1 contributes to leukocyte interactions with E-selectin, we injected anti-P-selectin mAb RB40.34 into venules of PSGL-1 $1^{-/-}$and $P S G L-1^{+/+}$mice (Figure 6b). The leukocyte rolling flux decreased from $12 \%$ in $P S G L-1^{+/+}$mice to $2.5 \%$ in PSGL-1/- mice, suggesting a major impairment in interactions of $P S G L-1^{-/}$leukocytes with E-selectin. The difference in rolling flux was not due to enhanced capture of WT leukocytes by binding of L-selectin to PSGL-1 (secondary tethering), because injection of anti-L-selectin mAb Mel-14 into PSGL-1/++ mice pretreated with anti-P-selectin mAb RB40.34 did not significantly decrease leukocyte rolling flux (Figure 6b). The difference in rolling flux was not due to plateletmediated delivery of WT leukocytes to endothelial cells (43), because flow cytometry established that RB40.34 circulated in sufficient concentrations to saturate all P-selectin sites on activated platelets for at least 24 hours after injection (data not shown). Taken together, these data confirm that E-selectin-mediated leukocyte capture (and hence rolling flux) is severely reduced in PSGL-1/-- mice. 
Table 2

Hemodynamic and microvascular parameters in TNF- $\alpha$-induced inflammation

Mouse genotype Mice Venules Diameter Centerline velocity Wall shear rate

$(n) \quad(n) \quad(\mu \mathrm{m}) \quad(\mu \mathrm{m} / \mathrm{s})$

PSGL-1-1

$10 \quad 42$

$33+1$

$2,800 \pm 200$

$34 \pm 1$

$2,900 \pm 300$

Diameter, centerline velocity, and wall shear rate are presented as mean \pm SEM of all investigated venules.

Injecting anti-P-selectin mAb RB40.34 into PSGL-1/-mice further decreased the rolling flux fraction from $7 \%$ to $2.5 \%(P<0.05)$ (Figure $6 \mathrm{c})$. This finding demonstrates that PSGL-1-independent ligands mediate leukocyte rolling on P-selectin. Addition of antiE-selectin mAb 9A9 eliminated the residual rolling, confirming that all rolling depended on P- and E-selectin (Figure 6c). Injecting only mAb 9A9 into $P S G L-1^{-1-}$ mice reduced the rolling flux fraction from $7 \%$ to $2.8 \%$ (Figure $6 \mathrm{c}$ ). The low rolling flux fraction observed in PSGL-1 $1^{-/-}$mice after treatment with $\mathrm{mAb}$ 9A9 reflects the severe but not complete impairment in P-selectin-mediated rolling. Addition of anti-P-selectin mAb RB40.34 elimi-nated this residual rolling, confirming the presence of PSGL-1-independent ligands for P-selectin.

To exclude potential nonspecific effects of the blocking Ab's used, we injected isotype-matched control mAb's (rat IgG1 for anti-P-selectin mAb RB40.34 and anti-E-selectin mAb 9A9; and rat IgG2a for antiL-selectin mAb Mel-14). In control mice pretreated with TNF- $\alpha$ for 2 hours to induce P- and E-selectin on endothelial cells, leukocyte rolling flux fraction was $19 \% \pm 3 \%$, which was not significantly altered by isotype control IgG1 $(22 \% \pm 2 \%)$. In control mice treated with TNF- $\alpha$ for 5 hours to induce L-selectin ligands on endothelial cells, and with mAb's RB40.34 and 9A9 to block P- and E-selectin, leukocyte rolling flux fraction was $9 \% \pm 3 \%$, which was not significantly altered by isotype control IgG2a $(8 \% \pm 0 \%)$.

Next, we investigated the distributions of leukocyte rolling velocities in TNF- $\alpha$-treated cremaster venules. PSGL-1 $1^{-/}$mice had a distribution of rolling velocities ( $V_{\text {avg }}, 8.7 \pm 0.4 \mu \mathrm{m} / \mathrm{s}$ ) that was similar to that of PSGL-1 ${ }^{+/+}$mice $\left(V_{\text {avg }}, 8.3 \pm 0.5 \mu \mathrm{m} / \mathrm{s}\right)$ (Figure $\left.7 \mathrm{a}\right)$. Treatment with anti-P-selectin mAb RB40.34 decreased rolling velocities to the same extent in both $P S G L-1^{-/-}$ mice $\left(V_{\text {avg }}, 6.0 \pm 0.3 \mu \mathrm{m} / \mathrm{s}\right)$ and $P S G L-1^{+/+}$mice $\left(V_{\text {avg }}\right.$, $6.3 \pm 0.3 \mu \mathrm{m} / \mathrm{s}$ ) (Figure $7 \mathrm{~b}$ ). Thus, fewer PSGL-1-/leukocytes roll on E-selectin, but the cells that interact roll at the same slow velocities as do PSGL-1/++ leukocytes. This demonstrates that PSGL-1 mediates tethering of leukocytes to E-selectin but does not contribute significantly to rolling after the cells tether.

In contrast, treatment with the anti-E-selectin $\mathrm{mAb}$ 9A9 dramatically increased leukocyte rolling velocities in PSGL-1 $1^{-/-}$mice $\left(V_{\text {avg }}, 64 \pm 5 \mu \mathrm{m} / \mathrm{s}\right)$, whereas it only slightly increased rolling velocities in $P S G L-1^{+/+}$mice $\left(V_{\text {avg }}, 16 \pm 1 \mu \mathrm{m} / \mathrm{s}, P<0.05\right)$ (Figure $7 \mathrm{c}$ ). These data con- $\left(\mathrm{s}^{-1}\right)$

$900 \pm 70$

$900 \pm 70$

firm that PSGL-1-independent ligands mediate rolling on P-selectin, but the rolling is much less stable than that mediated by PSGL-1.

\section{Discussion}

PSGL-1 binds to E-selectin, but there has been no definitive evidence that PSGL-1 contributes to leukocyte interactions with E-selectin under flow. Using independently generated $P S G L 1^{-1 /}$ mice, we demonstrate that PSGL-1 has an important function in tethering flowing leukocytes to E-selectin in vitro and in vivo. After the cells tether, however, PSGL-1 is not required to maintain slow rolling on E-selectin. These data reveal separable functions for adhesion molecules in supporting tethering and rolling of flowing cells.

In leukocyte lysates, PSGL-1 is one of the major glycoprotein ligands for E-selectin (10). Rolling of leukocytes on E-selectin correlates with expression of sLe -related epitopes on PSGL-1 (44-46). When immobilized on membranes, PSGL-1 or CD44 derived from hematopoietic progenitor cells supports rolling of E-selectin-expressing cells under flow (47). However, no previous study has addressed whether PSGL-1 or any other specific glycoprotein on intact
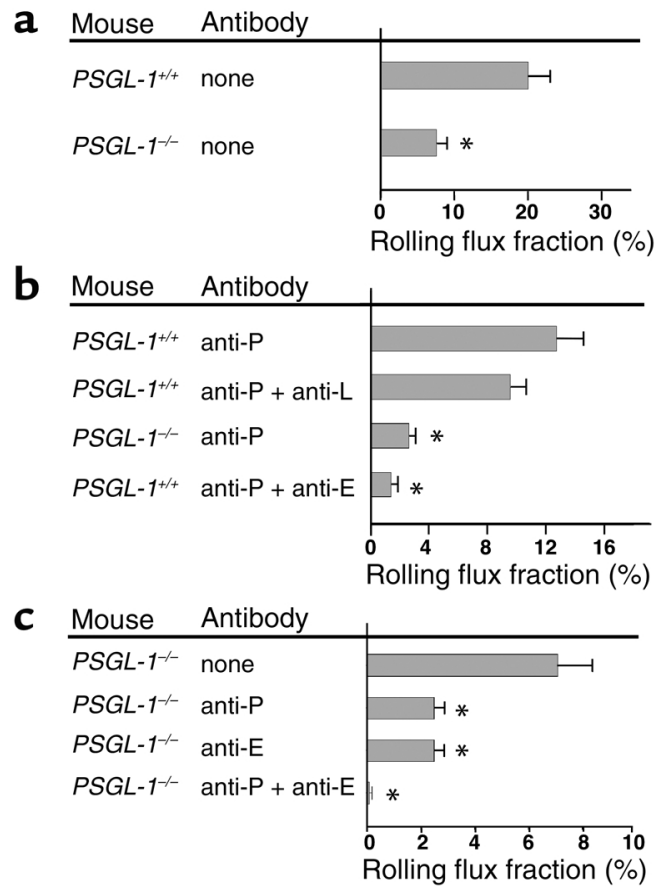

\section{Figure 6}

Leukocyte rolling flux fractions in venules of cremaster muscle from $P S G L-1^{+/+}$and PSGL-1-/- mice, subjected to TNF- $\alpha$-induced inflammation. Rolling flux fractions (mean \pm SEM) were measured 2-4 hours after injection of TNF- $\alpha$ in the presence or absence of anti-P-selectin mAb RB40.34, anti-E-selectin mAb 9A9, or anti$\mathrm{L}$-selectin $\mathrm{mAb}$ Mel-14. The asterisks indicate that the rolling flux fraction was significantly different from the reference value in the top bar of each panel, at $P<0.05$. 
a

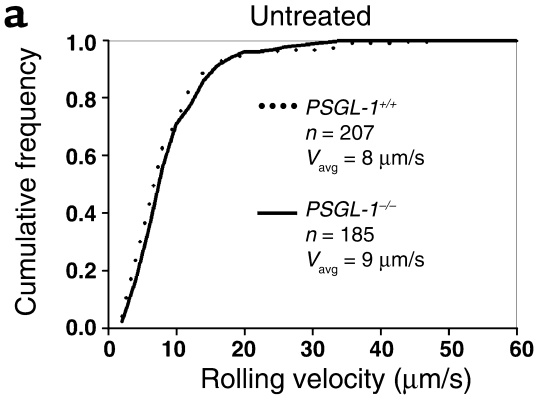

b

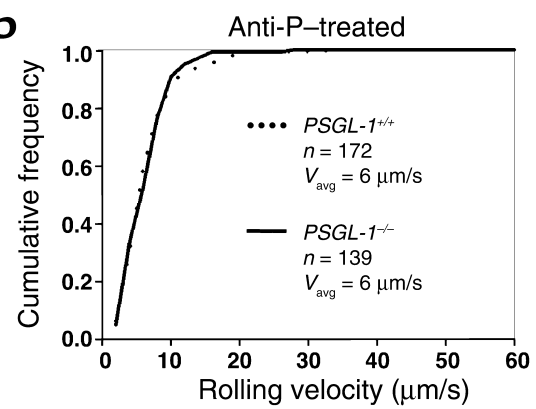

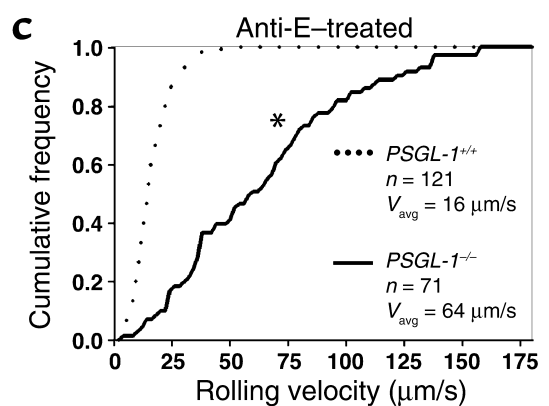

\section{Figure 7}

Leukocyte rolling velocity distributions in venules of cremaster muscle from PSGL-1+/+ and PSGL-1-1- mice, subjected to TNF- $\alpha$-induced inflammation. The cumulative histograms of rolling velocities of the indicated number of leukocytes are depicted. The mean rolling velocities $\left(V_{\text {avg }}\right)$ are also shown. (a) No mAb injected. (b) Injection of anti-P-selectin mAb RB40.34. (c) Injection of anti-E-selectin mAb 9A9. The asterisk indicates that the rolling velocities of $P S G L-1^{+/+}$and $P S G L-1^{-/-}$mice were significantly different, at $P<0.05$.

leukocytes supports tethering to or rolling on E-selectin under physiological flow conditions. Cells must present a ligand in sufficient density and with an optimal orientation that may reflect its length, its distribution on microvilli, and its clustering through molecular self-association or through interactions with cytoplasmic components $(3,48)$. An earlier study of PSGL-1-1- mice concluded that PSGL-1 is not required for leukocytes to roll on E-selectin in venules of TNF- $\alpha$-treated cremaster muscle, but the study did not specifically examine rolling on E-selectin when P-selectin function was blocked (22).

In vivo, we observed an approximately $80 \%$ reduction in rolling of PSGL-1-1- leukocytes on E-selectin in TNF- $\alpha$-activated venules when P-selectin was blocked, consistent with an approximately $70 \%$ reduction in binding of fluid-phase E-selectin-IgM to $P S G L-1^{-/-}$neutrophils. This establishes that PSGL-1 comprises a major subset of E-selectin ligands on the cell surface. In vitro, significantly fewer $P S G L-1^{-/-}$ leukocytes rolled on E-selectin under flow. This was due in part to decreased secondary tethering through loss of leukocyte-leukocyte interactions mediated by binding of L-selectin to PSGL-1. However, a major decrease in rolling leukocytes resulted from impaired primary tethering of flowing leukocytes to E-selectin. The PSGL-1/-- cells that successively tethered demonstrated rolling that was equivalent to that of $P S G L-1^{+/+}$ leukocytes, as measured by similar resistance to detachment and similar distributions of rolling velocities over a range of wall shear stresses. The tethering defect observed in vitro was even more severe in vivo. Compared with $P S G L-1^{+/+}$controls, far fewer PSGL-1/leukocytes rolled on E-selectin in venules of TNF- $\alpha$ treated cremaster muscle that were treated with an $\mathrm{mAb}$ to block P-selectin function. However, the residual cells that interacted with E-selectin rolled with slow velocities equivalent to velocities of $P S G L-1^{+/+}$ leukocytes. The decreased rolling flux reflected a major defect in primary tethering of PSGL-1/-- leukocytes to E-selectin, because the experiments were con- ducted in small and medium-sized venules where L-selectin-dependent leukocyte-leukocyte interactions are rare $(8,20,41)$. Furthermore, injection of an anti-L-selectin $\mathrm{mAb}$ in $P S G L-1^{+/+}$mice treated with anti-P-selectin $\mathrm{mAb}$ did not significantly reduce leukocyte rolling flux. Leukocyte-leukocyte interactions are more common in larger veins and in arteries where shear rates are higher (41). In these vessels, the number of $P S G L-1^{-/}$leukocytes rolling on E-selectin may be more severely decreased because of the loss of leukocyte tethers to other leukocytes through binding of L-selectin to PSGL-1.

Clustering of PSGL-1 on microvilli (11) and the extended length of the molecule (18) may enhance its ability to tether flowing leukocytes to E-selectin. This tethering function may explain the impaired recruitment of PSGL-1 $1^{-1-}$ Th1 cells to inflamed skin in a contact hypersensitivity model (49). The PSGL-1-independent ligands for E-selectin remain to be identified. One candidate is E-selectin ligand-1, an N-glycosylated protein that may oligomerize to enhance binding avidity, compensating for its relatively low binding affinity (50). The specific contributions of PSGL-1-mediated leukocyte tethering to E-selectin during inflammation require further study. Concurrent expression of P-selectin masks this function, because the interaction of P-selectin with PSGL-1 is an exceptionally effective mechanism for mediating leukocyte tethering (3). However, some inflammatory responses may induce expression of E-selectin but not P-selectin, particularly in humans (51-53).

Core-2 O-glycans are important components of several selectin ligands, including PSGL-1. There are three known core-2 $\beta 1,6-\mathrm{N}$-acetylglucosaminyltransferases (Core2GlcNAcTs) that create the core-2 branch on O-glycans (54-56). The major one of these transferases in leukocytes is Core2GlcNAcT-I (57). The defects in leukocyte rolling that we observed in venules of $P S G L-1^{-/-}$mice are similar to those observed in venules of Core2GlcNAcT-I ${ }^{-/-}$mice (20). In both

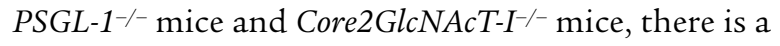


major reduction in leukocyte rolling in venules of TNF- $\alpha$-treated cremaster muscles that were injected with an mAb to block P-selectin function. In both mice, the residual leukocytes that tether to E-selectin are able to roll with the same slow velocities as those of WT mice. This suggests that PSGL- 1 is the primary Core2GlcNAcT-I-dependent tethering molecule for E-selectin on leukocytes. However, one study reported

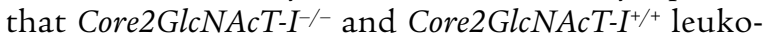
cytes roll equivalently on E-selectin in vitro (58). Another study reported that Core2GlcNAcT-I-I- leukocytes detach more readily from E-selectin in response to increasing wall shear stress (57). In contrast, we found that fewer PSGL-1 $1^{-/-}$leukocytes rolled on E-selectin in vitro, but these cells had shear resistance that was similar to that of $P S G L-1^{+/+}$leukocytes. Determining the relative contributions of Core2GlcNAcT-I and PSGL-1 to adhesive interactions with E-selectin will require direct comparisons of leukocytes from mice that lack either or both molecules. In vivo, the defect in tethering of Core2 GlcNAcT-I-I- leukocytes to E-selectin is particularly evident at higher shear rates (20). It will be interesting to determine if this is true for PSGL-1/- leukocytes.

We also found that ligands other than PSGL-1 mediate detectable but unstable leukocyte rolling on P-selectin in vitro and in vivo. PSGL-1 has specialized features that optimize its interactions with P-selectin under flow $(3,10)$. The PSGL-1-independent ligands on murine leukocytes must be limited in number and/or have very low affinity, because fluid-phase P-selectin-IgM did not detectably bind to PSGL-1/neutrophils. In vitro, PSGL-1 $1^{-/-}$leukocytes rolled only on very high densities of P-selectin, and even this rolling was modest and extremely unstable. By contrast, it was not difficult to detect rolling of PSGL-1/leukocytes on P-selectin in vivo, although the number of rolling cells was markedly reduced and the cells rolled significantly faster. In vivo, erythrocytes promote close proximity of circulating leukocytes with the endothelial cell surface, which may facilitate the interaction of PSGL-1-independent ligands with P-selectin $(59,60)$. The sensitivity of the PSGL-1-independent ligands to Pronase and OSGE implies that they are proteins, at least one of which may have clustered O-glycans. One candidate is CD24, a small glycosylphosphatidylinositol-linked protein that binds to P-selectin (24, 61-63). However, anti-CD24 mAb's, which inhibit most or all binding of purified CD24 to P-selectin, did not affect rolling of PSGL-1/-- leukocytes on P-selectin. Thus binding of a purified ligand to a selectin does not predict whether the ligand on a flowing leukocyte can mediate tethering to or rolling on a selectin.

In summary, we have identified a novel function for PSGL-1 as a ligand that tethers leukocytes to E-selectin in vitro and in vivo. We confirm that PSGL-1 supports adhesive interactions with $\mathrm{P}$ - and L-selectin. Thus, PSGL-1 contributes significantly to leukocyte adhesion to all three selectins under flow.

\section{Acknowledgments}

We thank Huaqin Pan and Bruce Roe for partial sequencing of the murine PSGL-1 gene, Bryan Buster for assistance with blood cell counts, Zoltan Laszik for tissue histology analysis, Tim Olson for preparation of Mel-14 F(ab')2 fragments, Patricia Clark and Catherine O'Connor for leukocyte differential measurements, Margaret Ober and Rowena Crittenden for microinjection of blastocysts, and Thomas Sato, Barry Wolitzky, Peter Altevogt, Dietmar Vestweber, and Paul Kincade for reagents. This work was supported by NIH grants HL-54502, AI-44902, HL-64381, and HL-54136. Lijun Xia was the recipient of a Beginning Grant-in-Aid award (9960321Z) from the Heartland Affiliate of the American Heart Association. Markus Sperandio was supported by the German Research Foundation (grant SP 621/1-1).

1. Springer, T.A. 1995. Traffic signals on endothelium for lymphocyte recirculation and leukocyte emigration. Annu. Rev. Physiol. 57:827-872.

2. Vestweber, D., and Blanks, J.E. 1999. Mechanisms that regulate the function of the selectins and their ligands. Physiol. Rev. 79:181-213.

3. McEver, R.P. 2001. Adhesive interactions of leukocytes, platelets, and the vessel wall during hemostasis and inflammation. Thromb. Haemost. 86:746-756.

4. Jung, U., and Ley, K. 1999. Mice lacking two or all three selectins demonstrate overlapping and distinct functions for each selectin. J. Immunol. 162:6755-6762.

5. Robinson, S.D., et al. 1999. Multiple, targeted deficiencies in selectins reveal a predominant role for P-selectin in leukocyte recruitment. Proc. Natl. Acad. Sci. USA. 96:11452-11457.

6. Ley, K., et al. 1995. Sequential contribution of L- and P-selectin to leukocyte rolling in vivo. J. Exp. Med. 181:669-675.

7.Jung, U., Ramos, C.L., Bullard, D.C., and Ley, K. 1998. Gene-targeted mice reveal importance of L-selectin-dependent rolling for neutrophil adhesion. Am. J. Physiol. 274:H1785-H1791.

8. Kunkel, E.J., and Ley, K. 1996. Distinct phenotype of E-selectin-deficient mice. E-selectin is required for slow leukocyte rolling in vivo. Circ. Res. 79:1196-1204.

9. Varki, A. 1997. Selectin ligands: will the real ones please stand up? J. Clin. Invest. 99:158-162.

10. McEver, R.P., and Cummings, R.D. 1997. Role of PSGL-1 binding to selectins in leukocyte recruitment. J. Clin. Invest. 100:485-492.

11. Moore, K.L., et al. 1995. P-selectin glycoprotein ligand-1 mediates rolling of human neutrophils on P-selectin. J. Cell Biol. 128:661-671.

12. Borges, E., et al. 1997. The P-selectin glycoprotein ligand-1 is important for recruitment of neutrophils into inflamed mouse peritoneum. Blood. 90:1934-1942.

13. Walcheck, B., Moore, K.L., McEver, R.P., and Kishimoto, T.K. 1996. Neutrophil-neutrophil interactions under hydrodynamic shear stress involve L-selectin and PSGL-1: a mechanism that amplifies initial leukocyte accumulation on P-selectin in vitro. J. Clin. Invest. 98:1081-1087.

14. Patel, K.D., Moore, K.L., Nollert, M.U., and McEver, R.P. 1995. Neutrophils use both shared and distinct mechanisms to adhere to selectins under static and flow conditions. J. Clin. Invest. 96:1887-1896.

15. Leppänen, A., et al. 1999. A novel glycosulfopeptide binds to P-selectin and inhibits leukocyte adhesion to P-selectin. J. Biol. Chem. 274:24838-24848.

16. Leppänen, A., White, S.P., Helin, J., McEver, R.P., and Cummings, R.D. 2000. Binding of glycosulfopeptides to P-selectin requires stereospecific contributions of individual tyrosine sulfate and sugar residues. J. Biol. Chem. 275:39569-39578.

17. Somers, W.S., Tang, J., Shaw, G.D., and Camphausen, R.T. 2000. Insights into the molecular basis of leukocyte tethering and rolling revealed by structures of P- and E-selectin bound to SLe(X) and PSGL-1. Cell. 103:467-479.

18. Li, F., et al. 1996. Post-translational modifications of recombinant P-selectin glycoprotein ligand-1 required for binding to P-and E-selectin. J. Biol. Chem. 271:3255-3264.

19. Norman, K.E., Moore, K.L., McEver, R.P., and Ley, K. 1995. Leukocyte rolling in vivo is mediated by P-selectin glycoprotein ligand-1. Blood. 86:4417-4421.

20. Sperandio, M., et al. 2001. Severe impairment of leukocyte rolling in venules of core 2 glucosaminyltransferase-deficient mice. Blood. 97:3812-3819.

21. Borges, E., et al. 1997. P-selectin glycoprotein ligand-1 (PSGL-1) on T 
helper 1 but not on T helper 2 cells binds to P-selectin and supports migration into inflamed skin. J. Exp. Med. 185:573-578.

22. Yang, J., et al. 1999. Targeted gene disruption demonstrates that P-selectin glycoprotein ligand 1 (PSGL-1) is required for P-selectin-mediated but not E-selectin-mediated neutrophil rolling and migration. J. Exp. Med. 190:1769-1782.

23. Yang, J., Galipeau, J., Kozak, C.A., Furie, B.C., and Furie, B. 1996. Mouse P-selectin glycoprotein ligand-1: molecular cloning, chromosomal localization, and expression of a functional P-selectin receptor. Blood. 87:4176-4186

24. Aigner, S., et al. 1995. Heat stable antigen (mouse CD24) supports myeloid cell binding to endothelial and platelet P-selectin. Int. Immunol. 7:1557-1565.

25. Maly, P., et al. 1996. The $\alpha(1,3)$ fucosyltransferase Fuc-TVII controls leukocyte trafficking through an essential role in L-, E-, and P-selectin ligand biosynthesis. Cell. 86:643-653.

26. Labow, M.A., et al. 1994. Characterization of E-selectin-deficient mice: demonstration of overlapping function of the endothelial selectins. Immunity. 1:709-720.

27. Ramachandran, V., et al. 1999. Tyrosine replacement in P-selectin glycoprotein ligand-1 affects distinct kinetic and mechanical properties of bonds with P- and L-selectin. Proc. Natl. Acad. Sci. USA. 96:13771-13776.

28. Alon, R., Fuhlbrigge, R.C., Finger, E.B., and Springer, T.A. 1996. Interactions through L-selectin between leukocytes and adherent leukocytes nucleate rolling adhesions on selectins and VCAM-1 in shear flow. J. Cell Biol. 135:849-865.

29. Hession, C., et al. 1992. Cloning of murine and rat vascular cell adhesion molecule-1. Biochem. Biophys. Res. Commun. 183:163-169.

30. Norman, K.E., et al. 2000. P-selectin glycoprotein ligand-1 supports rolling on E- and P-selectin in vivo. Blood. 96:3585-3591.

31. Lipowsky, H.H., and Zweifach, B.W. 1978. Application of the "two-slit" photometric technique to the measurement of microvascular volumetric flow rates. Microvasc. Res. 15:93-101.

32. Reneman, R.S., et al. 1992. Concentration and velocity profiles of blood cells in the microcirculation. In Advances in cardiovascular engineering. N.H.C. Hwang, V.T. Turitto, and M.R.T. Yen, editors. Plenum Press. New York, New York, USA. 25-50.

33. Veldman, G.M., et al. 1995. Genomic organization and chromosomal localization of the gene encoding human P-selectin glycoprotein ligand. J. Biol. Chem. 270:16470-16475.

34. Frenette, P.S., Mayadas, T.N., Rayburn, H., Hynes, R.O., and Wagner, D.D. 1996. Susceptibility to infection and altered hematopoiesis in mice deficient in both P- and E-selectins. Cell. 84:563-574.

35. Bullard, D.C., et al. 1996. Infectious susceptibility and severe deficiency of leukocyte rolling and recruitment in E-selectin and P-selectin double mutant mice. J. Exp. Med. 183:2329-2336.

36. Mayadas, T.N., Johnson, R.C., Rayburn, H., Hynes, R.O., and Wagner, D.D. 1993. Leukocyte rolling and extravasation are severely compromised in P selectin-deficient mice. Cell. 74:541-554.

37. Bullard, D.C., et al. 1995. P-selectin/ICAM-1 double mutant mice: acute emigration of neutrophils into the peritoneum is completely absent but is normal into pulmonary alveoli. J. Clin. Invest. 95:1782-1788.

38. Patel, K.D., and McEver, R.P. 1997. Comparison of tethering and rolling of eosinophils and neutrophils through selectins and P-selectin glycoprotein ligand-1. J. Immunol. 159:4555-4565

39. Norgard, K.E., et al. 1993. Characterization of a specific ligand for P-selectin on myeloid cells. A minor glycoprotein with sialylated O-linked oligosaccharides. J. Biol. Chem. 268:12764-12774.

40. Jung, U., and Ley, K. 1997. Regulation of E-selectin, P-selectin, and intercellular adhesion molecule 1 expression in mouse cremaster muscle vasculature. Microcirculation. 4:311-319.

41. Eriksson, E.E., Xie, X., Werr, J., Thoren, P., and Lindbom, L. 2001. Importance of primary capture and L-selectin-dependent secondary capture in leukocyte accumulation in inflammation and atherosclerosis in vivo. J. Exp. Med. 194:205-218.

42. Ley, K., and Gaehtgens, P. 1991. Endothelial, not hemodynamic, differ- ences are responsible for preferential leukocyte rolling in rat mesenteric venules. Circ. Res. 69:1034-1041.

43. Diacovo, T.G., Puri, K.D., Warnock, R.A., Springer, T.A., and Von Andrian, U.H. 1996. Platelet-mediated lymphocyte delivery to high endothelial venules. Science. 273:252-255.

44. Fuhlbrigge, R.C., Kieffer, J.D., Armerding, D., and Kupper, T.S. 1997. Cutaneous lymphocyte antigen is a specialized form of PSGL-1 expressed on skin-homing T cells. Nature. 389:978-981.

45. Borges, E., et al. 1997. The binding of T cell-expressed P-selectin glycoprotein ligand-1 to E- and P-selectin is differentially regulated. J. Biol. Chem. 272:28786-28792.

46. Kieffer,J.D., et al. 2001. Neutrophils, monocytes, and dendritic cells express the same specialized form of PSGL-1 as do skin-homing memory T cells: cutaneous lymphocyte antigen. Biochem. Biophys. Res. Commun. 285:577-587.

47. Dimitroff, C.J., Lee, J.Y., Rafii, S., Fuhlbrigge, R.C., and Sackstein, R. 2001. CD44 is a major E-selectin ligand on human hematopoietic progenitor cells. J. Cell Biol. 153:1277-1286.

48. Ramachandran, V., et al. 2001. Dimerization of a selectin and its ligand stabilizes cell rolling and enhances tether strength in shear flow. Proc. Natl. Acad. Sci. USA. 98:10166-10171.

49. Hirata, T., et al. 2000. P-selectin glycoprotein ligand 1 (PSGL-1) is a physiological ligand for E-selectin in mediating T helper 1 lymphocyte migration. J. Exp. Med. 192:1669-1675.

50. Wild, M.K., Huang, M.C., Schulze-Horsel, U., van Der Merwe, P.A., and Vestweber, D. 2001. Affinity, kinetics, and thermodynamics of E-selectin binding to E-selectin ligand-1. J. Biol. Chem. 276:31602-31612.

51. Pan, J., Xia, L., and McEver, R.P. 1998. Comparison of promoters for the murine and human P-selectin genes suggests species-specific and conserved mechanisms for transcriptional regulation in endothelial cells. J. Biol. Chem. 273:10058-10067.

52. Pan, J., Xia, L., Yao, L., and McEver, R.P. 1998. Tumor necrosis factor- $\alpha-$ or lipopolysaccharide-induced expression of the murine P-selectin gene in endothelial cells involves novel $\kappa \mathrm{B}$ sites and a variant ATF/CRE element. J. Biol. Chem. 273:10068-10077.

53. Yao, L., et al. 1999. Divergent inducible expression of P-selectin and E-selectin in mice and primates. Blood. 94:3820-3828.

54. Bierhuizen, M.F.A., and Fukuda, M. 1992. Expression cloning of a cDNA encoding UDP-GlcNAc:Gal $\beta 1-3-G a l N A c-R(G l c N A c$ to GalNAc) $\beta 1$ 6GlcNAc transferase by gene transfer into $\mathrm{CHO}$ cells expressing polyoma large tumor antigen. Proc. Natl. Acad. Sci. USA. 89:9326-9330.

55. Yeh, J.C., Ong, E., and Fukuda, M. 1999. Molecular cloning and expression of a novel beta-1, 6-N-acetylglucosaminyltransferase that forms core 2, core 4, and I branches. J. Biol. Chem. 274:3215-3221.

56. Schwientek, T., et al. 1999. Control of O-glycan branch formation. Molecular cloning of human cDNA encoding a novel beta1,6-N-acetylglucosaminyltransferase forming core 2 and core 4. J. Biol. Chem. 274:4504-4512.

57. Ellies, L.G., et al. 1998. Core 2 oligosaccharide biosynthesis distinguishes between selectin ligands essential for leukocyte homing and inflammation. Immunity. 9:881-890.

58. Snapp, K.R., et al. 2001. Differential requirements for the O-linked branching enzyme core $2 \beta 1-6-\mathrm{N}$-glucosaminyltransferase in biosynthesis of ligands for E-selectin and P-selectin. Blood. 97:3806-3811.

59. Nobis, U., Pries, A.R., Cokelet, G.R., and Gaehtgens, P. 1985. Radial distribution of white cells during blood flow in small tubes. Microvasc. Res. 29:295-304.

60. Schmid-Schonbein, G.W., Usami, S., Skalak, R., and Chien, S. 1980. The interaction of leukocytes and erythrocytes in capillary and postcapillary vessels. Microvasc. Res. 19:45-70.

61. Aigner, S., et al. 1997. CD24, a mucin-type glycoprotein, is a ligand for P-selectin on human tumor cells. Blood. 89:3385-3395.

62. Aigner, S., et al. 1998. CD24 mediates rolling of breast carcinoma cells on P-selectin. FASEB J. 12:1241-1251.

63. Friederichs, J., et al. 2000. The CD24/P-selectin binding pathway initiates lung arrest of human A125 adenocarcinoma cells. Cancer Res. 60:6714-6722. 